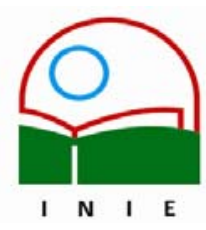

Universidad de Costa Rica

Facultad de Educación

Instituto de Investigación en Educación

ACTUALIDADES INVESTIGATIVAS EN EDUCACION

\title{
REACCIONES DE PROFESORAS Y PROFESORES DE LA UNIVERSIDAD DE COSTA RICA ANTE LA FLEXIBILIZACIÓN DEL CURRÍCULUM PARA ESTUDIANTES CON NECESIDADES EDUCATIVAS ESPECIALES
}

\section{Irma Arguedas Negrini ${ }^{1}$}

\begin{abstract}
RESUMEN: La Universidad de Costa Rica ha asumido el compromiso de garantizar la accesibilidad a los procesos de aprendizaje cuando esto sea requerido por estudiantes con necesidades educativas especiales. El presente trabajo se realizó con el propósito de recolectar información que favorezca que los procesos de igualdad de oportunidades se cumplan de la forma que aporte mayores beneficios para todos los involucrados y de proporcionar un espacio para que el personal docente manifestara sus reacciones ante sus experiencias con la flexibilización del currículum. Se analizan elementos que contribuyen a hacer la accesibilidad una realidad y se reportan las reacciones de diez profesoras $y$ profesores de la Sede Rodrigo Facio quienes han impartido cursos a estudiantes que han solicitado la aplicación del Artículo $37 \mathrm{del}$ Reglamento de Régimen Académico Estudiantil. Se concluye que la aplicación de adecuaciones curriculares o de acceso y la aceptación de la diversidad en el medio universitario pueden ser una fuente de aprendizaje y enriquecimiento.
\end{abstract}

Palabras clave: IGUALDAD DE OPORTUNIDADES/ ACCESIBILIDAD/ ADECUACIONES CURRICULARES EN LA UNIVERSIDAD DE COSTA RICA/ REACCIONES ANTE LA DIVERSIDAD/

ABSTRACT: At Universidad de Costa Rica efforts have been made to provide access to the curriculum to students with special needs. This work was done with the purpose of obtaining information that could help to make the provision of equal opportunities a process that benefits all who are involved, as well as to listen to the reactions of faculty who have applied accommodations for students that require them. Elements that contribute to make equal access a reality are analyzed, and the reactions to the experiences of ten educators from different areas of the university are reported. It is concluded that the provision of accommodations and the acceptance of student diversity at university settings could enhance the quality of teaching.

Key words: EQUAL OPPORTUNITIES/ ACCESIBILITY/ ACCOMMODATIONS AT UNIVERSIDAD DE COSTA RICA/ REACTIONS TO STUDENT DIVERSITYI

\section{Introducción}

El reconocimiento del derecho a la igualdad de oportunidades en diferentes ámbitos y la creciente valoración de la diversidad humana en nuestra sociedad, han tenido repercusiones en instituciones educativas de todos los niveles. Las Normas Uniformes para la Equiparación de Oportunidades de las Personas con Discapacidad, que fueron aprobadas por la Asamblea General de las Naciones Unidas en 1993, son pautas para que los estados adopten medidas que garanticen a las personas con discapacidad los mismos derechos y

\footnotetext{
1 Master of Science en Orientación Escolar, Department of Applied Behavioral Sciences in Education, Universidad Estatal de Oklahoma, E.E.U.U. Docente de la Escuela de Orientación y Educación Especial de la Universidad de Costa Rica.

Correo electrónico: quarco2000@yahoo.com
}

Artículo recibido: 6 de agosto, 2004

Aprobado: 8 de noviembre, 2004 
obligaciones que el resto de la sociedad. Dichas normas señalan importantes principios de responsabilidad, acción y cooperación (Jiménez, 1996). En Costa Rica, la aprobación por la Asamblea Legislativa en 1996 de la Ley 7600, Ley de Igualdad de Oportunidades para Personas con Discapacidad, representó un reto y a la vez la apertura de mayores posibilidades de desarrollo para la comunidad nacional.

La Universidad de Costa Rica ha asumido el compromiso de garantizar la igualdad de oportunidades de acceso en circunstancias equivalentes a estudiantes con discapacidad o con necesidades educativas especiales como una responsabilidad institucional permanente. Este compromiso es parte de los esfuerzos por ser una institución incluyente, en el marco de la construcción de una sociedad accesible para todos y con mayor tolerancia a la diversidad humana. El apoyo a estudiantes con discapacidad se ofrece desde los años setenta, aunque la primera formalización de servicios se dio en 1990 (Ruh y Stupp, 1994). Los servicios se han ofrecido mediante diversos programas. Actualmente, los brinda el Centro de Asesoría y Servicios para Estudiantes con Discapacidad (CASED), que pertenece a la Vicerrectoría de Vida Estudiantil. EI CASED trabaja en conjunto con los CASE (Centros de Asesoría Estudiantil) de las diferentes áreas en que está organizada la universidad (Artes y Letras, Ciencias Básicas, Ciencias Sociales, Ciencias Agroalimentarias, Ingenierías y Salud) en la promoción de la igualdad de oportunidades en el medio universitario a estudiantes con discapacidad (U. C. R., Vicerrectoría de Vida Estudiantil, 2002).

EI CASED ha sido concebido bajo el paradigma de la accesibilidad al entorno. La accesibilidad se considera "el requisito imprescindible para transformar los derechos en oportunidades reales" (Stupp, 2001, p. 138) y se define como "el proceso mediante el cual se potencian todas las áreas del individuo mediante el cambio de actitudes y de las adaptaciones necesarias, tomando en cuenta las necesidades personales, y con el fin de lograr una máxima funcionalidad y autonomía" (Echeverría, 1999, p. 12).

La ampliación del acceso para acoger a personas diversas implica múltiples desafíos para las instituciones educativas. En el caso específico de las profesoras y los profesores universitarios, el conocimiento de las características y necesidades de formación de sus estudiantes es indispensable para realizar la labor docente de forma efectiva. Dicho conocimiento incluye el comprender la interrelación entre los requerimientos de cada curso y las diversas formas en que cada estudiante aprende y entre las fortalezas y las dificultades 
de cada aprendiz. Cuando el acceso a los procesos de enseñanza y aprendizaje es limitado por no responder a las características, capacidades o estilos de aprendizaje de los estudiantes, se presenta una necesidad educativa especial. Las necesidades educativas especiales "forman parte de un continuum de necesidades educativas, que se extienden desde niveles ordinarios hasta necesidades muy significativas y que, ubicadas en el tiempo, podrían ser temporales o permanentes" (Zúñiga, 2002, p. 144). Un entorno accesible considera las necesidades de todas las personas, con o sin discapacidad (Montero, 2002).

El Artículo 37 del Reglamento de Régimen Académico Estudiantil (R. R. A. E.) se refiere a los medios para llevar a cabo adecuaciones de acceso o de currículum, cuando esto sea requerido por un estudiante con necesidades educativas especiales. Se trata de modificaciones en la planificación y de adaptaciones metodológicas que son llevadas a cabo por el personal docente cuando las características o dificultades de un alumno requieren de servicios de apoyo que le brinden igualdad de oportunidades para participar y aprender (U. C. R., Consejo Universitario, 2001). En el medio universitario las adecuaciones curriculares no implican cambios en los contenidos ni eliminación de cursos, sino reorganización de éstos de manera tal que se brinde acceso a los procesos de aprendizaje. Realizar adecuaciones es una forma de ser coherentes con la condición humana de diversidad sin disminuir los estándares de calidad. El Artículo 37 enfatiza en la formación de un equipo de apoyo que planifica la aplicación concreta de las adecuaciones de acceso o de currículum recomendadas por el CASED después de valorar cada caso. Al equipo le corresponde también el seguimiento y la evaluación de los procesos de adecuación y está formado por un docente de la carrera, la persona coordinadora de la carrera o directora de la unidad académica, una persona especialista en el área específica de discapacidad, un funcionario de la Vicerrectoría de Vida Estudiantil y la o el estudiante.

\section{Elementos que contribuyen a hacer la accesibilidad una realidad}

Múltiples experiencias han logrado identificar un conjunto de elementos que contribuyen a hacer realidad la práctica de la accesibilidad. Dentro del modelo de universidades accesibles para todos, se considera que el conocimiento acerca de los reglamentos específicos apoya el proceso de planificación y orienta las acciones (Stupp, 2001). Otro de los elementos es la práctica de la interdisciplinariedad, en la que la participación de la persona con discapacidad es esencial para realizar adaptaciones efectivas. La interdisciplinariedad se caracteriza por un intercambio de conocimientos y un enriquecimiento mutuo a nivel de destrezas, conceptos 
y actitudes. Es decir, es una práctica que fomenta el aporte de diversas disciplinas para el beneficio de todas y para el rompimiento de barreras con relación al acceso de los estudiantes a la educación superior (Montero y Hernández, 1978; Stupp, 2001). De esta forma, el equipo de apoyo, más que como un deber, puede verse como un instrumento que permite hacer el trabajo de forma eficaz. Las posibilidades de que esto sea así se ven aumentadas cuando las funciones de cada miembro son claras, se trabaja en un ambiente de respeto mutuo y se definen metas alcanzables.

Las reacciones que genera en las diferentes personas involucradas la flexibilización del currículum son muy variadas. Este es otro elemento crítico de la accesibilidad y uno de sus aspectos es la preparación del profesorado. El personal docente necesita actualización, reconocimiento de sus destrezas para enseñar y apoyo al adoptar un enfoque de enseñanza inclusivo. Tal enfoque se centra en métodos y estrategias que promueven el aprendizaje de todos los estudiantes, incorporan la práctica del acceso y anticipan las necesidades de los estudiantes (Shaw et al., 2001). La accesibilidad es un proceso complejo que implica transformaciones a nivel de actitudes, conocimiento y destrezas, tanto del profesorado como de la institución o sistema. Existen factores internos y externos que influyen en la percepción que adquieren los docentes de su trabajo con estudiantes con discapacidad. Dentro de los factores internos se encuentran actitudes y destrezas, conceptos en cuanto al valor de la diversidad y al desarrollo personal que puede significar un ambiente que favorece las posibilidades de logro para todos. Los factores externos tienen que ver con información, capacitación y facilidades de infraestructura con las que se cuente (Ballestero et al., 1997). Es importante contemplar las diferentes variables que intervienen, como el grado de dificultad de cada persona para enfrentar cambios o situaciones desconocidas, las experiencias previas, lo abrumadora que puede ser la cantidad de tareas y funciones del personal docente y los desacuerdos que puedan existir en cuanto a la forma de enfrentar las dificultades académicas de los estudiantes. Conocer acerca de este elemento crítico de la accesibilidad en el caso concreto de la Universidad de Costa Rica es una de las inquietudes que motivaron la realización del presente trabajo.

En algunos casos puede ser necesario que el profesor haga cambios metodológicos para atender las necesidades educativas de los estudiantes con discapacidad o con necesidades educativas especiales. Las instituciones que han incorporado la accesibilidad como uno de sus componentes ofrecen servicios de asesoría para llevar a cabo estos cambios, si el 
personal docente así lo requiere (Stupp, 2001). Dentro de los esfuerzos que las instituciones realizan para asegurar que los estudiantes con discapacidad tengan acceso a la educación, Beckman (2001) resalta el apoyo a los docentes para identificar sus necesidades de capacitación, para aprender a basarse en fortalezas y para conocer los perfiles de aprendizaje de los alumnos.

Las evaluaciones de las experiencias y percepciones de educadores realizadas por Salend (1999) indican que éstos tienen mayor disposición para hacer modificaciones cuando se dan las siguientes condiciones:

1- Reciben información concreta acerca del tiempo adicional que requiere la aplicación de los cambios

2- Cuentan con el conocimiento y la asesoría para aplicar las estrategias recomendadas

3- Los cambios recomendados son congruentes con la posición filosófica del educador

4- Valoran las modificaciones como justas, y

5- Las modificaciones benefician a otros estudiantes.

Friend y Bauwens (1988) agregan a esta lista de condiciones aquellas en que:

6- Los docentes continúan sintiéndose competentes como educadores, es decir, no sienten amenazadas sus capacidades

7- Se pueden identificar algunas ventajas que las modificaciones puedan implicar para los educadores

8- Las modificaciones se basan en las fortalezas del educador en el trabajo con estudiantes con y sin discapacidad, y

9- Se establecen mecanismos para intercambiar experiencias con otros docentes.

Se han hecho esfuerzos por determinar cuáles son algunas de las características ambientales y personales que contribuyen al logro de las metas de personas con necesidades educativas especiales y variadas situaciones de adversidad. Raskind et al. (1999) determinaron algunas variables que predicen el éxito en diversos ámbitos de la vida de personas con dificultades para el aprendizaje. Después de un seguimiento de 20 años, concluyeron que los atributos más relevantes son los seis que a continuación se definen: 
1- Conocimiento y comprensión de las características propias: Conocer las propias dificultades y considerarlas un aspecto más entre otros que definen a la persona.

2- Proactividad: Tener una participación activa en la toma de decisiones y expresar que se tiene el poder para hacer cambios en algunos aspectos de la vida personal o colectiva.

3- Perseverancia: Continuar los esfuerzos a pesar de las dificultades y expresar la actitud que las dificultades son necesarias para aprender.

4- Estabilidad emocional: Describir situaciones de manejo de estrés, tener buenas relaciones interpersonales y tolerar la frustración y la ambigüedad.

5- Establecimiento de metas realistas: Haber tenido metas en el pasado, tenerlas en el presente y hacer una planificación futura. Dichas metas son alcanzables y definidas de manera escalonada o por etapas.

6- Uso de sistemas de apoyo disponibles: Utilizar apoyos cuando se ofrecen o buscarlos cuando se necesitan.

Los estudios de Katz (1997) también resaltan la importancia de percibir a los estudiantes con una visión equilibrada de las capacidades y las dificultades, evitando un enfoque deficitario que limita el reconocimiento de los talentos y habilidades. Estos estudios confirman, además, el aporte de relaciones interpersonales respetuosas, de expectativas altas pero realistas y de oportunidades para la participación y la contribución. Por otra parte, la aceptación de las limitaciones se dificulta cuando en el medio se dan actitudes negativas hacia la discapacidad y la diversidad (Barga, 1996; Richie et al., 2003).

\section{Aplicación del Artículo 37 del Reglamento de Régimen Académico Estudiantil en la Universidad de Costa Rica}

El desarrollo de estrategias para la aplicación efectiva de la eliminación de barreras para el aprendizaje es determinante para cumplir con los planes concretos que se derivan de la normativa. Eliminar las barreras no sólo aumenta las posibilidades de permanencia y graduación de los estudiantes de educación superior, sino que es una fuente de aprendizaje para las instituciones. Con el propósito de que la igualdad de oportunidades se cumpla de manera que aporte mayores beneficios para los involucrados y de proporcionar un espacio para que profesoras y profesores de la Universidad de Costa Rica manifestaran sus reacciones ante las experiencias con la flexibilización del currículum, se programaron entrevistas con personal docente que ha impartido cursos a estudiantes de la Sede Rodrigo 
Facio acogidos al Artículo 37 del R. R. A. E. Se seleccionó una muestra intencional que incluyera a una profesora o un profesor de la Escuela de Estudios Generales y a representantes de diferentes facultades. Los participantes fueron seis profesoras y cuatro profesores pertenecientes a las Escuelas de Estudios Generales, de Artes Plásticas, de Lenguas Modernas, de Química, de Administración de Negocios, de Psicología, de Trabajo Social, de Ciencias de la Computación e Informática y del Programa de Tecnologías en Salud.

Se siguió una guía de entrevista elaborada conjuntamente con personal del CASED que incluye los elementos de la accesibilidad mencionados anteriormente y rescata algunos significados y enseñanzas obtenidos por los docentes a partir de su participación en procesos que promueven el acceso a la educación superior de estudiantes con discapacidad o con necesidades educativas especiales. Los aspectos específicos que se indagaron son:

- Lo que los profesores conocen acerca del Artículo 37 del R. R. A. E.

- Las necesidades educativas especiales que presentan los estudiantes.

- El tipo de participación que han tenido en lo equipos de apoyo.

- Las características de los equipos de apoyo que facilitan su funcionamiento.

- Las características de los equipos de apoyo que obstaculizan su funcionamiento.

- Los cambios metodológicos que han aplicado para atender las necesidades educativas de los estudiantes.

- Lo que ha significado para los docentes tener estudiantes que se han acogido al Artículo 37 del R. R. A. E.

- Las condiciones que necesita y los aportes que puede dar el profesor de un estudiante con necesidades educativas especiales.

- Las condiciones que necesita y los aportes que puede dar el estudiante con necesidades educativas especiales.

- Las sugerencias de los profesores para la aplicación de las adecuaciones.

\section{Experiencias de los profesores y sus equipos}

\subsection{Conocimiento del Artículo 37 del R. R. A. E:}

Siete de los diez profesores consultados conocen diversos aspectos de este artículo, como que es un derecho de los estudiantes, que se piden cambios en la metodología pero no en los contenidos de los cursos y que debe formarse un comité de apoyo. Ninguno menciona que el Artículo 37 especifica que el trabajo del comité de apoyo debe evaluarse. A pesar de 
esto, algunos profesores posteriormente relatan las estrategias que tienen sus equipos para evaluar.

\subsection{Tipo de necesidades educativas especiales que presentan los estudiantes:} Hacer los exámenes cortos de forma oral, modificar las tareas y tener una mayor comunicación con el estudiante para llevar un seguimiento más detallado de su rendimiento, son algunas de las adecuaciones que se han aplicado, por ejemplo, con un joven con autismo. Varios profesores tienen estudiantes con déficit de atención y/o dificultades específicas para el aprendizaje. En estos casos se solicitan adecuaciones como proporcionar más tiempo para los exámenes, dividir asignaciones o evaluaciones en tractos y establecer estrategias para lograr mayor retención de la información. Los estudiantes con discapacidad visual tienen adecuaciones como utilizar grabadoras y software especial, realizar exámenes de forma oral o con modificación en el tamaño y la intensidad de la letra, contar con el apoyo de tutores y con acceso a materiales transcritos a Braille. Los estudiantes sordos tienen el apoyo de intérpretes de LESCO, el acceso a tecnología computarizada, tutorías y apoyo en toma de apuntes. Tres de los profesores tienen estudiantes con discapacidades físicas. Estos requieren de más tiempo para algunas actividades, algunas modificaciones en las técnicas, en el caso de una joven que es estudiante de una carrera artística y apoyo para comer y movilizarse por parte de un acompañante, en el caso de una joven con parálisis cerebral infantil. Un profesor tenía (se retiraron de la carrera) dos estudiantes que requerían tiempo adicional para algunas actividades cuando, debido a sus trastornos mentales, aumentaban sus dificultades.

Los estudiantes de la carrera de Terapia Física que tienen discapacidad visual necesitan un mayor desarrollo de la capacidad para palpar; el fin con ellos, así como con estudiantes de otras carreras, es que lleguen a tener la misma autonomía que los otros compañeros. Aproximadamente la mitad de los profesores expresan el interés de tener más información acerca de las características de los estudiantes, tanto de las dificultades como de los talentos y fortalezas.

\subsection{Participación de los profesores en los equipos de apoyo:}

Hay algunos casos en los que no se ha cumplido con la formación de equipos de apoyo. Tres de los profesores coordinan sólo con el estudiante después de recibir la carta que indica que el estudiante requiere alguna adecuación. Una profesora recibió la carta varias semanas 
después de haber iniciado el semestre y no pudo darle atención especial a la estudiante porque ésta se retiró del curso. Los cuatro profesores que se encuentran en esa situación manifiestan que les gustaría contar con las opiniones y asesoría de otros en un equipo de apoyo.

Los otros profesores entrevistados se consideran miembros activos de los equipos de apoyo, en los que cada integrante aporta desde su campo. Han podido unificar criterios en cuanto a la forma de trabajar y de adaptarse a la presencia de intérpretes o acompañantes. Dan opiniones como "ha sido un equipo equilibrado", "sentí la satisfacción de colaborar como una pieza más", "hemos tenido mucho apoyo y capacitación del CASE y del CASED", "funciona perfecto pero no podría hacerlo sin apoyo".

Una profesora coordinadora de uno de los equipos, entrena a otros profesores y planea las adecuaciones que se requieren para los cursos de los semestres venideros, todo en estrecha colaboración con una especialista del CASED. Los entrevistados consideran que, en términos generales, ha habido mucha anuencia y apertura, sin embargo han enfrentado algunas pocas situaciones de resistencia por parte de otros docentes. Otra profesora, aunque dispuesta a aplicar adecuaciones, manifiesta estar en desacuerdo con algunos aspectos. Cree que los estudiantes pueden perjudicarse si justifican sus hábitos ineficaces con el hecho de tener dificultades. Otro manifiesta que no ha sido difícil porque siempre ha dado atención individual, le ha interesado el desarrollo de la creatividad de cada estudiante y ha trabajado con grupos pequeños, debido a su área de especialidad. Sin embargo, ha necesitado más asesoría de la que ha recibido. Una profesora considera que la metodología modular adoptada por su unidad académica ha resultado adecuada para implementar adecuaciones cuando algún estudiante lo requiere.

\subsection{Características de los equipos de apoyo que facilitan la aplicación de las adecuaciones:}

Los profesores valoran positivamente lo siguiente:

- Compromiso, esfuerzo adicional e información sobre sí mismo por parte del estudiante.

- Oferta de soluciones a los profesores por parte del equipo. Aceptar que se necesita invertir en tiempo y capacitación. 
- Asesoría del CASED y los CASE para aclarar dudas, ofrecer información a compañeros de los estudiantes con adecuación cuando se considera necesario y para capacitar a los asistentes de los cursos.

- La aclaración que hacer adecuaciones no significa disminuir las exigencias ni cambiar los contenidos de los cursos.

- Aceptación en los cursos de la presencia de acompañantes cuando las personas con discapacidad así lo requieren.

- Panorama claro y amplio de las dificultades y las fortalezas de los estudiantes que han solicitado la aplicación del Artículo 37 del R. R. A. E.

- Apoyo y capacitación a los profesores cuando tienen temor de aplicar adecuaciones.

- Adecuada anticipación para reunirse como equipo y para solicitar servicios que toman tiempo, como las transcripciones a Braille de materiales escritos en letra regular.

- Utilización de las destrezas y conocimientos de los diferentes miembros del equipo.

- Comunicación efectiva durante la implementación y habilidad para tomar acuerdos mutuos.

Estas características describen un enfoque de trabajo que enfatiza la importancia del apoyo especializado y el compromiso, de la visión integral de las personas, de la búsqueda de soluciones que sean aceptables para todos los involucrados y de la potenciación del recurso humano, que hacen posible la accesibilidad a la educación superior a estudiantes con discapacidad o con necesidades educativas especiales.

\subsection{Características de los equipos de apoyo que obstaculizan la aplicación de las adecuaciones:}

- Insuficiente conocimiento de las valoraciones que se han hecho de cada estudiante.

- Que el estudiante no estudie, falte a lecciones o no sea abierto en cuanto a sus necesidades.

- Insuficiente preparación de los profesores en aspectos metodológicos.

- Desacuerdo por parte de algunos profesores en cuanto a que los estudiantes tienen diversas formas de aprender.

- Vaguedad en algunas de las recomendaciones proporcionadas por los especialistas en discapacidad.

- Carencia de información en cuanto a la forma de relacionarse con personas con discapacidad y otras características, por ejemplo, personas con deficiencias visuales o que presentan tics. 
El origen de estos obstáculos parece girar en torno a la insuficiencia de conocimientos técnicos y apoyo y actitudes personales basadas en juicios previos infundados. Además, algunos equipos de apoyo tienen limitaciones presupuestarias relacionadas con la carestía de equipos como los de electroterapia o dificultades para recibir con rapidez materiales escritos en Braille.

\subsection{Cambios metodológicos efectuados:}

En términos generales, los profesores encuentran pocas diferencias en las estrategias de enseñanza que utilizan con las personas que han solicitado la aplicación del Artículo 37 del R. R. A. E. y las que utilizan con el resto de los estudiantes. La mayoría refiere no sentirse complicados por realizar adecuaciones curriculares porque han usado variedad de estrategias siempre. Acostumbran consultar a especialistas cuando esos recursos están disponibles. La mayoría brinda más apoyo verbal y está dispuesta a hacer exámenes orales. Establecen mayor contacto con los estudiantes para determinar si las adecuaciones están resultando funcionales y para estimularlos. Adicionalmente, los observan con más detenimiento durante las lecciones y al hacer exámenes. Con la excepción de un profesor, que dedica bastante tiempo cada semana a la coordinación del equipo de apoyo, los docentes entrevistados creen que el tiempo adicional que se requiere no es significativo; lo consideran parte del trabajo de un educador. Las modificaciones más frecuentes son la utilización de tiempo extra para los exámenes y la división de la materia en varias pruebas más cortas en lugar de en pocas extensas. En cursos de laboratorio se han hecho modificaciones, a veces en los equipos que se utilizan, otras en la manera en que los estudiantes elaboran informes o en el trabajo con los asistentes y compañeros.

La opinión de los profesores es que brindarles condiciones a los estudiantes con discapacidad no ha sido muy diferente de brindarles condiciones a los estudiantes que no tienen discapacidad. Señalan también que algunas de las estrategias que han aprendido benefician la enseñanza de todas las personas, presenten o no necesidades educativas especiales, tomando en consideración que no todas las estrategias sirven en todos los casos.

\subsection{Significado de la experiencia para los docentes:}

Todas las personas entrevistadas manifiestan que la experiencia ha sido sumamente enriquecedora. Consideran que las ha ayudado a ser mejores docentes, que les ha 
permitido tomar conciencia de las diferencias individuales y, en algunos casos, reconocer a otros estudiantes que no se han identificado por medio del CASED. Les da satisfacción apoyar y contribuir a que los alumnos reciban un trato que favorece su formación integral. La mayoría empezó con temor y el aprendizaje ha sido grande. No niegan que han enfrentado un reto, pero creen que ha sido una experiencia muy interesante. Han aprendido de los estudiantes con discapacidad, de sus actitudes. Compartir con personas con discapacidad los ha hecho más sensibles a lo que dicen y hacen. Un profesor comenta que ha aprendido a aceptar que él también tiene dificultades y no siempre puede llevar a cabo experiencias de enseñanza-aprendizaje de forma exitosa con todos los estudiantes. Ha representado una oportunidad para aceptar sus limitaciones en lugar de creer que los problemas son sólo de los otros. Valoran el haber aprendido que no todos los estudiantes funcionan de igual manera, que cada uno tiene su propio ritmo, tenga o no discapacidad. Agregan que algunos de los estudiantes con discapacidad han servido como un ejemplo y han tenido un efecto motivador con sus compañeros.

\subsection{Las condiciones que necesita el profesor para la aplicación de adecuaciones:}

A lo que más se refieren las personas entrevistadas es a la necesidad de información de todo tipo: de las limitaciones, discapacidades, fortalezas y otras características de los estudiantes, de estrategias metodológicas para las diferentes situaciones y de la legislación vigente. Creen que sería beneficioso aprovechar la experiencia de profesores de otras áreas. Los que no han tenido asesoría directa por parte de especialistas del CASED desean tener una o dos reuniones por semestre en las que puedan consultar acerca de dificultades que se presentan en los cursos.

Los docentes manifiestan que necesitan información previa, apoyo de un equipo, aprovechamiento de la experiencia producto del trabajo con personas sin discapacidad y comunicación abierta con el estudiante. Una profesora considera que es conveniente, tanto para el profesor como para el estudiante, que en el medio se acepte la idea que la persona con discapacidad se beneficia si tiene el máximo posible de independencia. Agrega que los compañeros de los estudiantes con discapacidad también necesitan asesoría y ha observado que tienden a tener comportamientos extremos: o los protegen de forma excesiva o los aíslan. 


\subsection{Las condiciones que necesita y el aporte que puede dar el estudiante que se acoge al Artículo 37 del R. R. A. E:}

Los profesores entrevistados consideran que los estudiantes pueden tener mayores logros si:

- Tienen profesores exigentes que les piden responsabilidad.

- Han desarrollado destrezas desde la primaria y la secundaria, que los ayudan a compensar sus limitaciones.

- Poseen un buen nivel de autoconocimiento. Conocen sus dificultades, explican en qué consisten sus necesidades educativas especiales y cómo se manejan, aclaran cuando alguna de las estrategias es perjudicial para ellos.

- Son perseverantes, esforzados y estudian para enriquecerse y no solo para los exámenes.

- No se sienten inferiores, sino diferentes.

- Les gusta y tienen aptitud para la carrera que han elegido.

- Son autónomos, tienen la actitud de dar lo mejor y no esperan que se disminuyan las exigencias.

- Las adecuaciones los ayudan a superarse en lugar de estancarlos.

- Tienen acceso a material y a tecnología.

- Cuentan con profesores que los comprenden y les exigen pero les dan oportunidades de nivelarse.

- Son abiertos a aceptar lo que pueden y no pueden hacer, se atreven a experimentar, son creativos y originales. Aceptan críticas.

- Tienen igualdad de oportunidades pero también igualdad de requisitos.

Algunos profesores opinan que hay estudiantes que no han aprovechado el apoyo que se les ha ofrecido. Sería pertinente analizar cada uno de los casos, para determinar las posibles causas y soluciones. El ingreso y permanencia en una institución de educación superior supone enfrentar múltiples desafíos para una persona con discapacidad o alguna necesidad educativa especial; el aprovechamiento de las oportunidades que brinda la accesibilidad requiere de mucha persistencia por parte de los estudiantes. Lock y Layton (2001) han encontrado que también es importante que cada estudiante conozca sus fortalezas y debilidades específicas en relación con su desempeño académico. La persona tendrá un mejor funcionamiento general si este conocimiento propio en el área del aprendizaje se acompaña de lo que Higgins et al. (2002) han denominado compartamentalización, que 
significa visualizar las dificultades como un elemento más dentro del conjunto general de características personales.

\subsection{Sugerencias adicionales aportadas por los profesores:}

Para la etapa de planeamiento del proceso, los profesores entrevistados sugieren que la primera reunión se efectúe antes que empiece cada ciclo lectivo, para así tomar en cuenta las necesidades de los estudiantes desde el principio. La mayoría insiste en que necesitan más información sobre las diferentes limitaciones que presentan los estudiantes. Una profesora y un profesor sugieren que desde la etapa de planeamiento se les indique los aspectos que deben tomarse en cuenta en las evaluaciones de cada caso, tanto formativas como finales. Agregan que en esta etapa se pueden solicitar las direcciones electrónicas de los miembros de los equipos y otros colaboradores para que reciban información pertinente (metodología, artículos) y se lleve a cabo un intercambio de opiniones. El interés por intercambiar ideas con otros profesores se manifestó repetidas veces. Algunos mencionan la importancia de la coordinación con las instituciones de educación secundaria para que la accesibilidad se estructure de forma continua. Una profesora opina que las condiciones de accesibilidad deben brindarse también para los estudiantes de posgrado, lo que es congruente con este enfoque de continuidad en las condiciones de acceso.

Las personas que han recibido asesoría directa, por parte de especialistas del CASED o los CASE, consideran que ésta es determinante durante la implementación. La necesidad de información acerca de las necesidades educativas de los estudiantes, así como la de apoyo en la parte metodológica se vuelve a mencionar para esta etapa. Apuntan que enriquecer sus recursos metodológicos favorece el aprendizaje de todos los estudiantes. Algunos docentes se quejan de la tardanza con que les han llegado las solicitudes. Otros manifiestan que desean mayor especificidad en las adecuaciones requeridas; por ejemplo, a veces sólo se solicita más tiempo y desean mayor información, tanto acerca de otros requerimientos como de si la solicitud de más tiempo se puede ir modificando, a lo largo del semestre, de común acuerdo con el estudiante u otros miembros del equipo. Los equipos que tienen un buen nivel de funcionamiento reportan haber tenido que solucionar algunos conflictos. La capacidad para enfrenar conflictos de manera constructiva cobra relevancia aquí.

A pesar de que algunos de los equipos están en constante evaluación de los procesos de adecuación, ninguno de los profesores mencionó que la responsabilidad de evaluar está 
estipulada en el Artículo 37 del R. R. A. E. La mayoría de los profesores no ha participado en evaluaciones finales de la aplicación de adecuaciones en sus cursos. Una profesora expresa no haber participado en evaluaciones, excepto cuando el estudiante ha perdido un curso. Otros sugieren un formulario que permita reportar la experiencia, intercambiar ideas de metodología y hacer recomendaciones. También manifiestan que lo que se va a evaluar debe anticiparse desde el planeamiento, para ir guardando la información. Una profesora dice que su inquietud más grande es el seguimiento que se da al desarrollo integral del estudiante porque opina que una de las metas en el caso de estudiantes con discapacidad o con necesidades educativas especiales debe ser que mejore las herramientas para ser independiente y capaz de compensar sus dificultades en la medida de lo posible. Uno de los profesores, cuyo equipo está formado nada más por él y la estudiante, se sentiría apoyado por las opiniones de otros profesores; ha tenido casos en que ha quedado con dudas de si lo que adaptó realmente sirvió. Una profesora aporta que se debe evaluar si lo que se solicita como adecuación es realmente necesario, ya que ha observado situaciones en las que se solicita más tiempo para una prueba cuando podría ser mejor adecuar la forma de hacer la prueba propiamente. Tres de los profesores reportan ser miembros de equipos muy estructurados. Realizan evaluaciones formativas y finales con el apoyo del CASED, los CASE y los tutores. La profesora de Terapia Física enfatiza en las evaluaciones los logros de los estudiantes en cuanto a autonomía y los déficits que les han quedado por falta de equipo; trata de anticiparse a las necesidades de semestres próximos para que el equipo esté preparado. Una profesora sugiere la asignación de horas al profesor cuando los planes de adecuación requieran una cantidad de tiempo significativa.

\section{Conclusiones}

La aplicación de adecuaciones curriculares o de acceso en el medio universitario puede constituirse en una parte integrante del quehacer docente y de la institución en su conjunto. Estos procesos pueden fomentar el desarrollo, tanto a nivel de actitudes y metodología como a nivel institucional y para el beneficio de todos, no sólo de la población con discapacidad. El apoyo a la diversidad es fuente de aprendizaje y enriquecimiento. Muchas de las medidas que promueven el acceso de los estudiantes con discapacidad o con necesidades educativas especiales se llevan a cabo satisfactoriamente. Contribuye a esto que los miembros de los equipos de apoyo han aceptado las modificaciones por convencimiento y que las estrategias sugeridas han sido tolerables y realistas. La experiencia de las profesoras y los profesores entrevistados ejemplifica cómo, a pesar de ser 
un desafío, estos procesos pueden convertirse en enriquecedoras experiencias de aprendizaje en las que los involucrados desarrollan destrezas que disminuyen el impacto de las situaciones adversas.

Se encontró anuencia por parte de los profesores a modificar la manera en que enseñan, sobre todo cuando las adecuaciones se perciben como justas. No obstante, manifiestan repetidamente que no siempre se sienten suficientemente preparados para adaptar las estrategias a las necesidades individuales de algunos estudiantes, independientemente de que presenten alguna discapacidad. Tanto el sistema institucional como el trabajo de los diferentes miembros de los equipos de apoyo han sido efectivos en los esfuerzos por brindar acceso no discriminatorio a la educación superior. El papel de personal especializado como el de los CASE y del CASED ha sido determinante, así como los aportes por parte de los estudiantes. Cuando el acceso se acompaña de persistencia, conocimiento de las características propias, independencia y apertura, los resultados son positivos.

La capacitación, el apoyo y la información brindados a los docentes han aumentado la seguridad al aplicar las adecuaciones. Sin embargo, se ha resaltado la importancia de conocer más a fondo las fortalezas y las necesidades de formación tanto de los profesores como de los estudiantes. Es necesario continuar con las medidas que han favorecido la aplicación del Artículo 37 del R. R. A. E., así como fortalecer algunos aspectos; es decir, el programa puede seguir evolucionando, basándose en las necesidades de la población y en la clarificación del papel que deben y pueden tener las docentes y los docentes, el personal especializado, el personal de apoyo y la misma institución en facilitar el acceso a una educación con altos estándares de calidad para todos.

\section{Recomendaciones}

6.1. A pesar de que se ha logrado una buena difusión del Artículo 37 del R. R. A. E., es necesario enfatizar el aspecto de la evaluación para que los procesos sean más completos y se puedan sistematizar las experiencias.

6.2. Los profesores necesitan apoyo para enfrentar las diversas implicaciones de los procesos de adecuación. Algunas de las implicaciones son: que el tiempo adicional que se requiere sea considerable, la presencia de intérpretes o asistentes en el aula y la necesidad de mayor información relacionada con la razón de las adaptaciones y la forma de satisfacerlas, incluyendo el conocimiento y utilización de fortalezas. Los profesores necesitan asesoría para implementar diversas estrategias, lo que beneficia a la totalidad de los 
estudiantes y no sólo a los que tienen discapacidad o presentan alguna necesidad educativa especial. En este sentido, es necesario continuar con la asesoría de los especialistas del CASED y otros miembros de los equipos en cuanto a estrategias metodológicas, la adaptación de equipos y materiales y la capacitación de asistentes y compañeros de los cursos.

6.3. El esfuerzo para resolver situaciones complejas se maximiza cuando se aprovecha la contribución de puntos de vista variados. El trabajo interdisciplinario, en el que la solución de problemas es colaborativa, es lo más indicado en la dinámica de trabajo de los grupos de apoyo que se constituyen para llevar a cabo las adecuaciones que requieren estudiantes con discapacidad o con necesidades educativas especiales.

6.4. La presencia de las características de los equipos de apoyo, que según los profesores entrevistados facilitan la aplicación de adecuaciones, puede valorarse en cada grupo de trabajo.

6.5. Algunas medidas pueden tomarse para disminuir la presencia de características de los grupos de apoyo que obstaculizan la aplicación efectiva de las adecuaciones.

6.6. Lo que los profesores opinan acerca de las condiciones que necesitan, así como el aporte que pueden dar los estudiantes a quienes se les aplican adecuaciones curriculares 0 de acceso, tiene mucha similitud con la información que han arrojado otros estudios y debe tenerse presente en cada uno de los equipos de apoyo.

6.7. Otro aspecto que puede llevar a mayores logros es estrechar la vinculación entre diferentes componentes del sistema educativo, en este caso entre instituciones de educación secundaria e instituciones de educación superior. El conjunto de condiciones ambientales, actitudes y comportamientos que los estudiantes requieren debe fomentarse de manera sistemática lo más temprano posible en la vida de los estudiantes.

\section{Agradecimientos}

El trabajo se pudo realizar gracias a la anuencia de las profesoras y los profesores que fueron entrevistados. Se agradecen la colaboración del personal del CASED y de los CASE de la Sede Rodrigo Facio en el diseño de la guía de entrevista y la localización de participantes, así como las atinadas sugerencias de la Lic. Carmen Cubero durante la elaboración del manuscrito. 


\section{Referencias}

Asamblea Legislativa; Asesoría Presidencial para los Asuntos de la Población con Discapacidad (1996). Ley 7600 de Igualdad de oportunidades para las personas con discapacidad en Costa Rica. Heredia, Costa Rica: Consejo Nacional de Rehabilitación y Educación Especial.

Ballestero, J.; Fernández, W.; Picado, E.; Sánchez, R. (1997). El Departamento de Orientación de las Instituciones Educativas de Secundaria como Asesor para facilitar la Educación de los Estudiantes con Discapacidad en el aula regular. Tesis de Licenciatura, Universidad de Costa Rica, Costa Rica.

Barga, N. (1996). Students with Learning Disabilities in Education: Managing a Disability. Journal of Learning Disabilities. 29 (4): 413-421.

Beckman, P. (2001). Access to the General Education Curriculum for Students with Disabilities. Recuperado el 12 de agosto de 2003, de http://www.ericfacility.net/ericdigests/ed458735.html.

Echeverría, A. (1999). Acceso de las personas con Discapacidad a la Educación Superior: Universidad de Costa Rica. Tesis de Maestría, Universidad de Costa Rica, Costa Rica.

Friend, M.; Bauwens, J. (1988) Managing Resistance: An Essential Consulting Skill for Learning Disabilities Teachers. Journal of Learning Disabilities. 21, (9): 556-561.

Higgins, E.; Raskind, M.; Goldberg, R.; Herman, K. (2002). Stages of Acceptance of a Learning Disability: The Impact of Labeling. Learning Disabilities Quarterly. 25 (Winter 2002): 3-18.

Katz, M. (1997). On Playing a Poor Hand Well. Insights from the Lives of Those Who Have Overcome Childhood Risks and Adversities. New York, U.S.A.: W. W. Norton \& Company.

Jiménez, R. (1996). Los derechos humanos de las personas con discapacidad. San José, Costa Rica: ILANUD/ Programa Mujer, Justicia y Género/ Instituto Interamericano de Derechos Humanos/ Disabled Peoples' International.

Lock, R.; Layton, C. (2001). Succeeding in Postsecondary Ed Through Self-Advocacy. Teaching Exceptional Children. 34 (2): 66-71.

Montero, C. (2002). Accesibilidad al Entorno en los Centros de Educación Superior. En Las personas con discapacidad en la educación superior. Una propuesta para la diversidad y la igualdad. San José, Costa Rica: Fundación Justicia y Género. pp.111123.

Montero, L.; Hernández, A. (1978). Aprendizaje e interdisciplinariedad. Programa de especialización del profesorado de e.g.b. Madrid, España: Universidad Nacional de Educación a Distancia, Ministerio de Educación y Ciencia. 
Raskind, M.; Goldberg, R.; Higgins, E.; Herman, K. (1999). Patterns of Change and Predictors of Success in Individuals with Learning Disabilities: Results From a Twenty-Year Longitudinal Study. Learning Disabilities Research \& Practice. 14 (1): 35-49.

Richie, B.; Ferguson, A.; Gomez, M.; El-Khoury, D.; Adamaly, Z. (2003). Resilience in Survivors of Traumatic Limb Loss. Disability Studies Quarterly. 23 (2): 29-41.

Ruh, P.; Stupp, R. (1994). Mitos y realidades del compromiso de la Universidad de Costa Rica con las personas con discapacidad. En Proyecto de Universidad para el Siglo XXI: Cátedra Emma Gamboa. San José: Oficina de Publicaciones de la Universidad de Costa Rica. pp. 403-408.

Salend, S. (1999). So What's with Our Inclusion Program? Evaluating Educators' Experiences and Perceptions. Teaching Exceptional Children. 32 ( 2): 46-54.

Shaw, S.; Scott, S.; McGuire, J. (2001). Teaching College Students with Learning Disabilities. Recuperado el 12 de agosto de 2003, de http://www.ericfacility.net/ericdigests/ed459548.html.

Stupp, R. (2001). Universidades accesibles para todos. Revista Educación. 25 (2): 137-145.

Universidad de Costa Rica, Consejo Universitario. (2001). Reglamento de Régimen Académico Estudiantil. Ciudad Universitaria Rodrigo Facio, San José: Oficina de Publicaciones de la Universidad de Costa Rica.

Universidad de Costa Rica, Vicerrectoría de Vida Estudiantil, Oficina de Orientación. (2002). Antecedentes, generalidades y funciones del Centro de Asesoría y Servicios a Estudiantes con Discapacidad. San José, Costa Rica. (Documento mimeografiado).

Werner, E.; Smith, R. (2001). Journeys from Childhood to Midlife. Risk, Resilience, and Recovery. New York, U.S.A.: Cornell University Press.

Zúñiga, E. (2002). Las Adecuaciones Curriculares en la universidad: Un Derecho y una Responsabilidad Impostergables. En Las personas con discapacidad en la educación superior. Una propuesta para la diversidad y la igualdad. San José: Fundación Justicia y Género. pp. 141-153. 